\title{
Efficient circuit-level modelling of ballistic CNT using piecewise non-linear approximation of mobile charge density
}

\author{
Tom J Kazmierski, Dafeng Zhou and Bashir M Al-Hashimi \\ School of Electronics and Computer Science, University of Southampton, Southampton, SO17 1BJ, UK \\ tjk,dz05r,bmah@ecs.soton.ac.uk
}

\begin{abstract}
This paper presents a new carbon nanotube transistor (CNT) modelling technique which is based on an efficient numerical piece-wise non-linear approximation of the non-equilibrium mobile charge density. The technique facilitates the solution of the self-consistent voltage equation in a carbon nanotube such that the CNT drain-source current evaluation is accelerated by more than three orders of magnitude while maintaining high modelling accuracy. The model is currently limited to ballistic transport but can be extended to non-ballistic modes of transport when a suitable theory is developed while researchers study phenomena that sometimes prevent electrons in a carbon nanotube from going ballistic. Our results show that while the accuracy and speed of the proposed model vary with the number of piece-wise segments in the mobile charge approximation, it is possible to obtain a speed-up of more than 1000 times while maintaining the accuracy within less than $2 \%$ in terms of average RMS error compared with the state of the art theoretical reference CNT model implemented in FETToy. This numerical efficiency makes our model particularly suitable for implementation in circuit-level, eg. SPICE-like, simulators where large numbers of such devices may be used to build complex circuits.
\end{abstract}

\section{INTRODUCTION}

Following the recent advancements in the understading of Carbon Nanotube Transistor (CNT) operation [1], [2], several models have been developed [3], [4], [5], [6], [7], [8] for implementation in SPICE-like simulators to enable simulations of future analog and digital systems built with CNT devices. The standard modelling technique is to derive the total CNT drain-source current from some approximation of the nonequilibrium mobile charge injected into the nanotube when a voltage is applied between the drain and the source [1]. The main stumbling block in the development of a circuitlevel model is the fact that accurate calculation of the mobile charge involves numerical integration of the densities of states over the number of allowed energy levels using the Fermi probability distribution. In addition, as the total drain current is affected not only by the non-equilibrium mobile charge in the nanotube but also by the charges present at terminal capacitances, the solution of an implicit non-linear algebraic equation is necessary using some iterative approach, such as the Newton-Raphson method, to calculate the drain current. For example, the MATLAB script named FETToy [9] available on line as a reference implementation of the state-of-the art ballistic CNT theory, requires more than 12 seconds of the CPU time on a Pentium IV PC to calculate a family of current drain characteristics for a single transistor [10]. The numerical complexity inherent in the physical model is impractical in simulations of circuits that might involve very large numbers of CNT devices. Very recently techniques have been proposed to eliminate the need for costly Newton-Raphson iterations and the numerical evaluation of the Fermi-Dirac integral while still maintaining a good agreement with the physical theory [8], [10]. They are based on piece-wise approximation of the charge density profiles, either linear [8] or non-linear [10] to simplify calculations. In this paper we generalise the piecewise non-linear approach we proposed earlier [10] and develop a more accurate technique where linear, quadratic and thirdorder polynomials are used to maintain an accuracy better than $2 \%$ in terms of average RMS error. This compares favourably with the $5 \%$ error that was reported for the simple linear approximation [8], and we still achieve a speed up of more than three orders of magnitude compared with FETToy. The salient feature of the presented approximation technique is the high modelling accuracy while enabling a fast numerical solution of the self-consistent voltage equation using a simple algorithm, without the need for CPU intensive Newton-Raphson iterations and evaluation of state densities from Fermi-Dirac probability distribution integrals.

\section{Mobile ChARGE DENSITY AND SELF-CONSISTENT VOLTAGE}

When an electric field is applied between the drain and the source of a CNT, a non-equilibrium mobile charge is induced in the nanotube[1], [11], [12]:

$$
\Delta Q=q\left(N_{S}+N_{D}-N_{0}\right)
$$

where $N_{S}$ is the density of positive velocity states filled by the source, $N_{D}$ is the density of negative velocity states filled by the drain and $N_{0}$ is the equilibrium electron density. These densities are determined by the Fermi-Dirac probability distribution as follows: 


$$
\begin{aligned}
N_{S} & =\frac{1}{2} \int_{-\infty}^{+\infty} D(E) f\left(E-U_{S F}\right) d E \\
N_{D} & =\frac{1}{2} \int_{-\infty}^{+\infty} D(E) f\left(E-U_{D F}\right) d E \\
N_{0} & =\int_{-\infty}^{+\infty} D(E) f\left(E-E_{F}\right) d E
\end{aligned}
$$

where $U_{S F}$ and $U_{D F}$ are defined as

$$
\begin{gathered}
U_{S F}=E_{F}-q V_{S C} \\
U_{D F}=E_{F}-q V_{S C}-q V_{D S}
\end{gathered}
$$

$V_{S C}$ is the self-consistent voltage, a recently introduced concept [1] which illustrates that the CNT energy band is affected by external terminal voltages, $D(E)$ is the density of states, $E_{F}$ is the Fermi level, $f$ is the Fermi probability distribution, $q$ is the electronic charge and $E$ represents the energy levels per nanotube unit length. The self-consistent voltage $V_{S C}$ is implicitly related to the device terminal voltages and charges at terminal capacitances by the following non-linear algebraic equation [1], [8]:

$$
V_{S C}=-\frac{Q_{t}+q N_{S}\left(V_{S C}\right)+q N_{D}\left(V_{S C}\right)+q N_{0}}{C_{\Sigma}}
$$

where $Q_{t}$ represents the charge stored in terminal capacitances and is defined as

$$
Q_{t}=V_{G} C_{G}+V_{D} C_{D}+V_{S} C_{S}
$$

where $C_{G}, C_{D}, C_{S}$ are the gate, drain, and source capacitances correspondingly and the total terminal capacitance $C_{\Sigma}$ is

$$
C_{\Sigma}=C_{G}+C_{D}+C_{S}
$$

The standard approach to the solution of equation (7) is to use the Newton-Raphson iterative method and in each iteration evaluate the integrals in equations (3) and (4) to obtain the state densities $N_{D}$ and $N_{S}$.

\section{CIRCUIT MODEL}

In the earlier work [10] we proposed to apportion equal parts of the equilibrium mobile charge density $N_{0}$ to the drain and source. This facilitates circuit implementation of the model because now the corresponding non-equilibrium mobile charge densities $Q_{S}$ and $Q_{D}$ can be modelled as nonlinear circuit capacitances connected between a conceptual inner node, which represents the self-consistent potential, and CNT terminal nodes:

$$
Q_{S}\left(V_{S C}\right)=q\left(N_{S}-\frac{1}{2} N_{0}\right)
$$

and

$$
Q_{D}\left(V_{S C}\right)=q\left(N_{D}-\frac{1}{2} N_{0}\right)
$$

The resulting equivalent circuit is shown in figure 1 where $\Sigma$ is the hypothetical inner node described above, which comprises all the CNT charges.

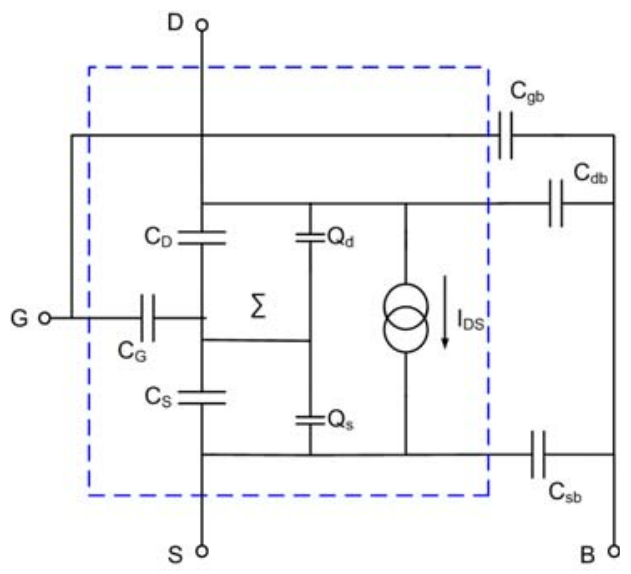

Fig. 1. Equivalent circuit for the proposed CNT model.

According to the ballistic CNT transport theory [1], [9] the drain current caused by the transport of the non-equilibrium charge across the nanotube can be calculated using the FermiDirac statistics as follows:

$$
I_{D S}=\frac{2 q k T}{\pi \hbar}\left[\mathcal{F}_{0}\left(\frac{U_{S F}}{k T}\right)-\mathcal{F}_{0}\left(\frac{U_{D F}}{k T}\right)\right]
$$

where $\mathcal{F}_{0}$ represents the Fermi-Dirac integral of order $0, k$ is Boltzmann's constant, $T$ is the temperature and $\hbar$ is reduced Planck's constant.

If the self-consistent voltage $V_{S C}$ is known, the evaluation of the drain current poses no numerical difficulty as energy levels $U_{S F}, U_{D F}$ can be found quickly from equations (5), (6). Then $I_{D S}$ can be calculated directly from equation (12) which uses the closed-form analytical solution of the FermiDirac integral of order 0 [8]:

$$
\mathcal{F}_{0}(\eta)=\log \left(1+e^{\eta}\right)
$$

However, as it has already been highlighted above, the solution of the self-consistent voltage equation (7) is very time consuming because it involves a Newton-Raphson iterative process in which each iteration requires numerical integration to obtain state densities $N_{D}\left(V_{S C}\right)$ and $N_{D}\left(V_{S C}\right)$. The next section outlines a piece-wise non-linear approximation technique that eliminates the need for these complex calculations while maintaining a high modelling accuracy.

\section{PROPOSED PIECEWISE NON-LINEAR APPROXIMATION OF MOBILE CHARGE DENSITY}

We have developed and investigated two accurate piecewise approximations of the non-equilibrium mobile charge density dependence on the self-consistent voltage $V_{S C}$ at the 
source and drain. The boundaries between regions could be determined symbolically from physical parameters [8]. Here we employ a purely numerical, rather than symbolic, approach where the boundaries are calculated to minimise the RMS deviation from the theoretical curves. Polynomial equations for regions were then obtained according to the same rule while assuring the continuity of the first derivative, over the temperature range $150 \mathrm{~K} \leq T \leq 450 \mathrm{~K}$ and Fermi level range $-0.5 \mathrm{eV} \leq E_{F} \leq 0 \mathrm{~V}$. The approximations were compared with the theoretical curves calculated from equations (3), (10) and (4), (11) correspondingly. Model 1, illustrated in figure 2 uses three piecewise regions: 1) linear, when $V_{S C} \leq \frac{E_{F}}{q}-$ $0.08 \mathrm{~V}, 2)$ quadratic, when $\frac{E_{F}}{q}-0.08 \mathrm{~V}<V_{S C}<\frac{E_{F}}{q}+0.08 \mathrm{~V}$ and 3) zero, when $V_{S C} \geq \frac{E_{F}}{q}+0.08 \mathrm{~V}$. The more accurate Model 2, uses four piecewise regions as shown in figure 3: 1) linear, when $V_{S C} \leq \frac{E_{F}}{q}-0.28 \mathrm{~V}$, 2) quadratic, when $\frac{E_{F}}{q}-0.28 \mathrm{~V}<V_{S C} \leq \frac{q}{E_{F}}-0.03 \mathrm{~V}$, 3) 3rd order, when $\frac{E_{F}}{q}-0.03 V<V_{S C} \leq \frac{E_{F}}{q}+0.12 V$ and 3) zero, when $V_{S C}>\frac{E_{F}}{q}+0.12 V$.

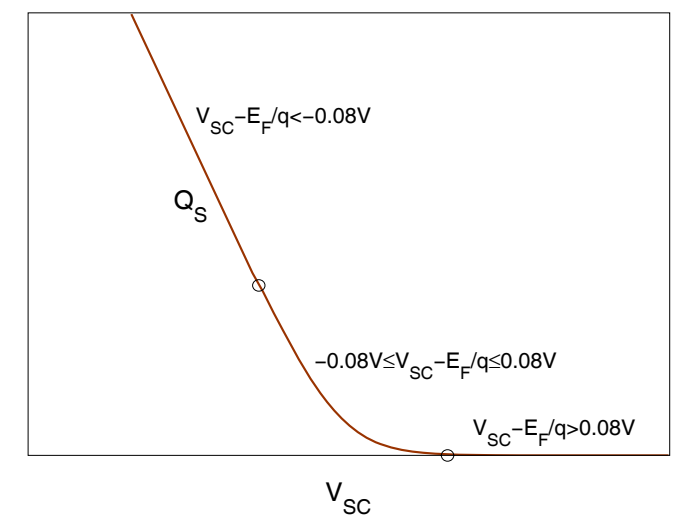

Fig. 2. Model 1: three-piece non-linear approximation of mobile charge.

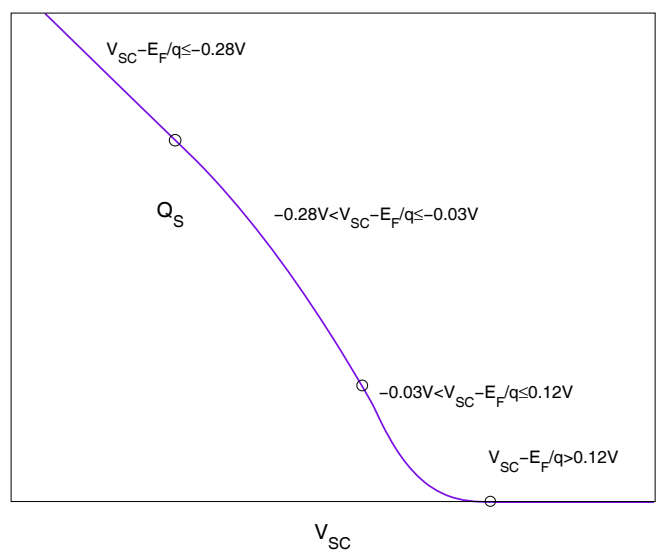

Fig. 3. Model 2: four-piece non-linear approximation of mobile charge.

The piece-wise non-linear approximations for the drain and source charge densities calculated using Model 1 for the typical temperature and Fermi level values of $T=300 \mathrm{~K}$ and $E_{F}=-0.32 \mathrm{eV}$ correspondingly are illustrated and compared with the theoretical graphs in figure 4. A similar illustration and comparison for Model 2 is shown in figure 5. It is clear, from figures 4 and 5, that Model 2 reflects more accurately the mobile charge densities especially in the ranges representing larger values of the charge. It is possible to use more sections for an even higher accuracy but at some computational expense. We are currently investigating in more detail how the number of sections affects the trade-off between accuracy and speed.

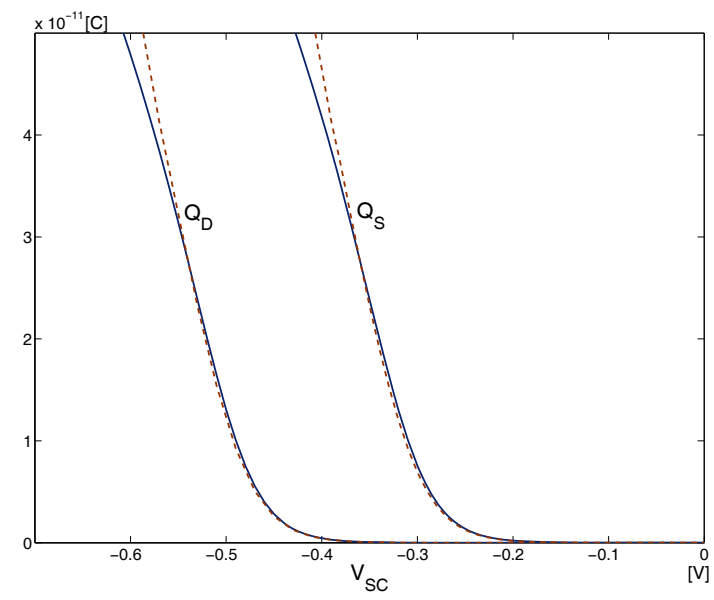

Fig. 4. Mobile charge densities at drain and source for $T=300 \mathrm{~K}$ and $E_{F}=-0.32 \mathrm{eV}$ (solid lines) and their piece-wise approximations using Model 1 (dashed lines).

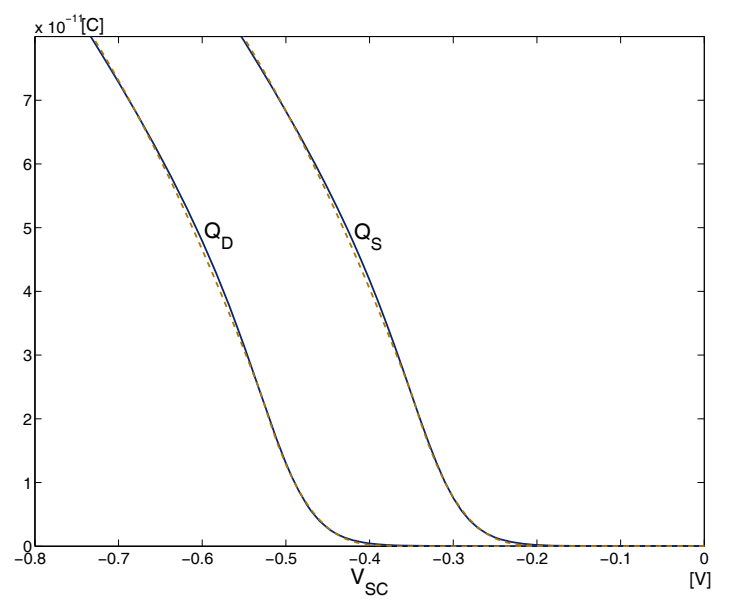

Fig. 5. Mobile charge densities at drain and source for $T=300 \mathrm{~K}$ and $E_{F}=-0.32 \mathrm{eV}$ (solid lines) and their piece-wise approximations using Model 2 (dashed lines).

Both models use polynomials of order not greater than 3 and hence allow a closed-form solution of the self-consistent voltage equation (7) thus completely eliminating the need for Newton-Raphson iterations and numerical evaluation of state density integrals. The optimised non-linear approximation 
outlined above ensures an accuracy of about $2 \%$ for the fourpiece model compared with the theoretical model of CNT ballistic transport [1] and represents an improvement over the piecewise linear symbolic approximation [8]. This purely numerical approach not only gives the model developer control over the approximation accuracy but also leads to a significant saving in the processing time as shown in the next section.

\section{DRAIN CURRENT CALCULATION}

Once the self-consistent voltage $V_{S C}$ is calculated from the closed-form solutions of equation (7), whose approximation contains only linear, quadratic or 3rd order polynomial terms, the total drain current can be directly obtained from equations (5), (6), (12) and (13):

$I_{D S}=\frac{2 q k T}{\pi \hbar}\left[\log \left(1+e^{\frac{E_{F}-q V_{S C}}{k T}}\right)-\log \left(1+e^{\frac{E_{F}-q\left(V_{S C}-V_{D S}\right)}{k T}}\right)\right.$

These calculations are extremely fast, as Newton-Raphson iterations and integration of the Fermi-Dirac probability distribution are now eliminated. CPU times and $I_{D S}$ prediction accuracy for both models were measured against the theoretical model [1] implemented in FETToy [9]. Table I shows average CPU times for both models and those for FETToy. For accurate measurement, a number of calculations were carried out by invoking all models 5, 10, 50 and 100 times. Results show that both models are more than three orders of magnitude faster than FETToy: Model 1 is about 3400 times faster and Model 2 - more than 1100 times. The extent to which the modelling accuracy was compromised by numerical approximation was also measured by calculating average RMS errors in the simulations. Figures 6 and 7 show the $I_{D S}$ characteristics calculated by FETToy compared with both Model 1 and Model 2 respectively. Table II shows average RMS error for both models. As expected, Model 2 is slightly more accurate with errors not exceeding $2 \%$ at $T=300 \mathrm{~K}$ and $E_{F}=-0.32 \mathrm{eV}$ throughout the typical ranges of drain voltages $V_{D S}$ and gate bias $V_{G}$.

TABLE I

AVERAGE CPU TIME COMPARISON

\begin{tabular}{c|c|c|c}
\hline Loops & FETToy & Model 1 & Model 2 \\
\hline 5 & $64.43 \mathrm{Sec}$ & $0.02 \mathrm{Sec}$ & $0.06 \mathrm{Sec}$ \\
\hline 10 & $128.78 \mathrm{Sec}$ & $0.04 \mathrm{Sec}$ & $0.12 \mathrm{Sec}$ \\
\hline 50 & $642.44 \mathrm{Sec}$ & $0.19 \mathrm{Sec}$ & $0.56 \mathrm{Sec}$ \\
\hline 100 & $1287.45 \mathrm{Sec}$ & $0.38 \mathrm{Sec}$ & $1.12 \mathrm{Sec}$ \\
\hline
\end{tabular}

Figures 6 and 7 show the $I_{D S}$ characteristics calculated by FETToy compared with both Model 1 and Model 2 respectively. As shown in Table II, both models maintain a high accuracy in terms of the average RMS error. As expected, Model 2 is slightly more accurate with errors not exceeding $2 \%$ at $T=300 \mathrm{~K}$ and $E_{F}=-0.32 \mathrm{eV}$ throughout the typical ranges of drain voltages $V_{D S}$ and gate bias $V_{G}$.

In addition, drain current characteristics were calculated and compared for accuracy with the theoretical CNT model in

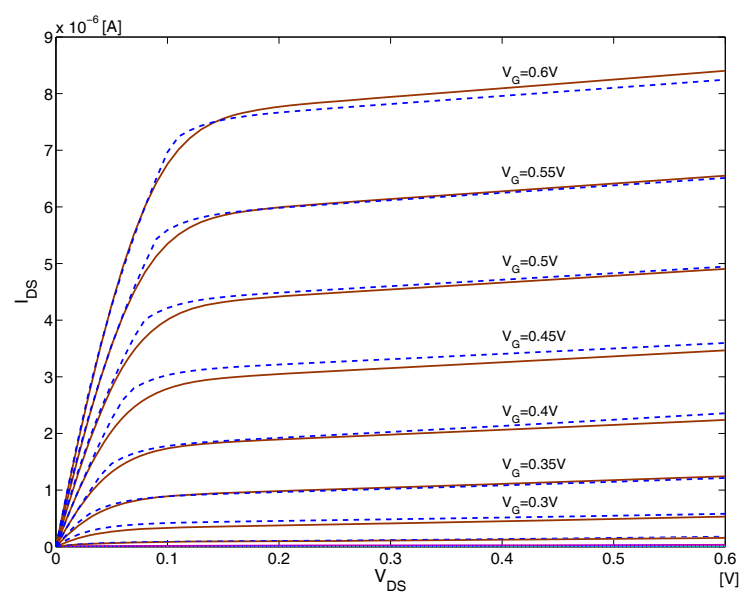

Fig. 6. Drain current characteristics at $T=300 \mathrm{~K}$ and $E_{F}=-0.32 \mathrm{eV}$ for FETToy(solid lines) and piece-wise approximation using Model 1 (dashed lines)

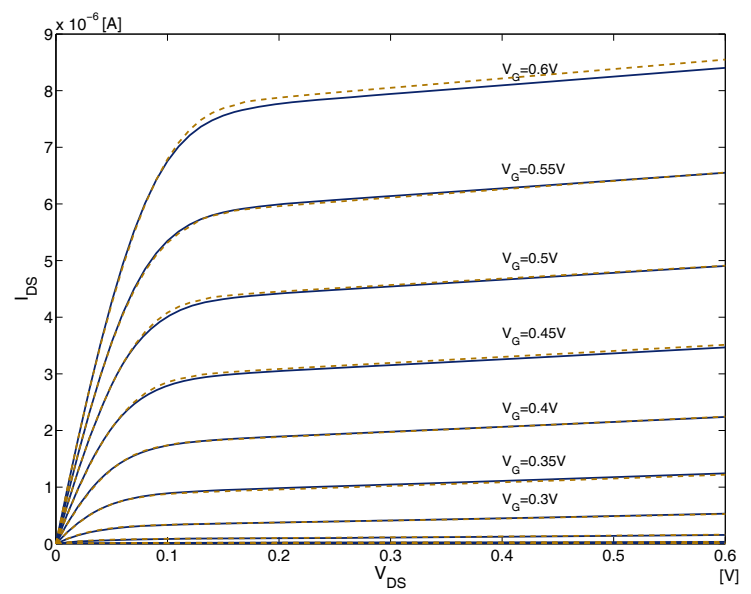

Fig. 7. Drain current characteristics at $T=300 \mathrm{~K}$ and $E_{F}=-0.32 \mathrm{eV}$ for FETToy(solid lines) and piece-wise approximation using Model 2 (dashed lines).

FETToy using different temperature and Fermi level values. Tables II, III and IV show average RMS errors for both models at temperatures $T=150 \mathrm{~K}, 300 \mathrm{~K}, 450 \mathrm{~K}$ and Fermi levels $E_{F}=-0.5 \mathrm{eV},-0.32 \mathrm{eV},-0.5 \mathrm{eV}$. Figures 8 and 9 illustrate the drain current characteristics of Model 2 at different temperatures and Fermi levels.

\section{COMPARISON WITH EXPERIMENTAL RESULTS}

Additionally, to validate the performance of the proposed modelling technique, both Model 1 and Model 2 were compared with recently published experimental results [13] for an n-type carbon nanotube transistor fabricated with K-doping and grounded back gate. The transistor's parameters were: $\mathrm{d}=1.6 \mathrm{~nm}, t_{o x}=50 \mathrm{~nm}$ and $E_{F}=-0.05 \mathrm{eV}$ and measurements were taken at the temperature $\mathrm{T}=300 \mathrm{~K}$. Figures 10 and 11 show that the proposed models, as well as the theoretical ballistic model implemented in FETToy, maintain good accuracy compared 
TABLE V

AVERAGE RMS ERRORS IN $I_{D S}$ COMPARISON TO THE EXPERIMENTAL RESULTS OF FETTOY MODEL AND THE PROPOSED MODELS FOR $d=1.6 \mathrm{~nm}, t_{o x}=50 \mathrm{~nm}, \mathrm{~T}=300 \mathrm{~K}$ AND $E_{F}=-0.05 \mathrm{eV}$.

\begin{tabular}{|l|c|c|c|}
\hline$V_{G}[V]$ & FETToy & Model 1 & Model 2 \\
\hline \hline 0.2 & $8.5 \%$ & $10.7 \%$ & $9.9 \%$ \\
\hline 0.4 & $7.8 \%$ & $9.8 \%$ & $8.9 \%$ \\
\hline 0.6 & $7.2 \%$ & $9.3 \%$ & $8.1 \%$ \\
\hline
\end{tabular}

with the experimental measurements. Table $\mathrm{V}$ shows that corresponding average RMS errors do not exceed $10 \%$.

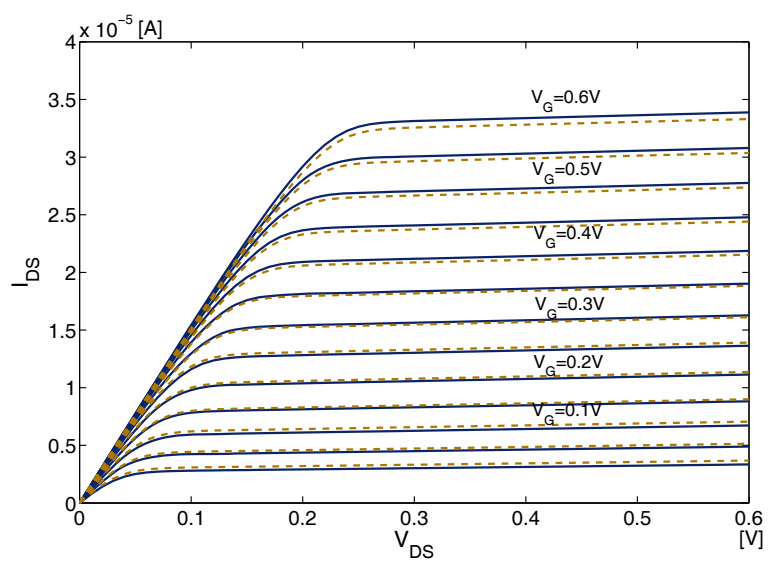

Fig. 8. Drain current characteristics at $T=150 \mathrm{~K}$ and $E_{F}=0 \mathrm{eV}$ for FETToy(solid lines) and piece-wise approximation using Model 2 (dashed lines).

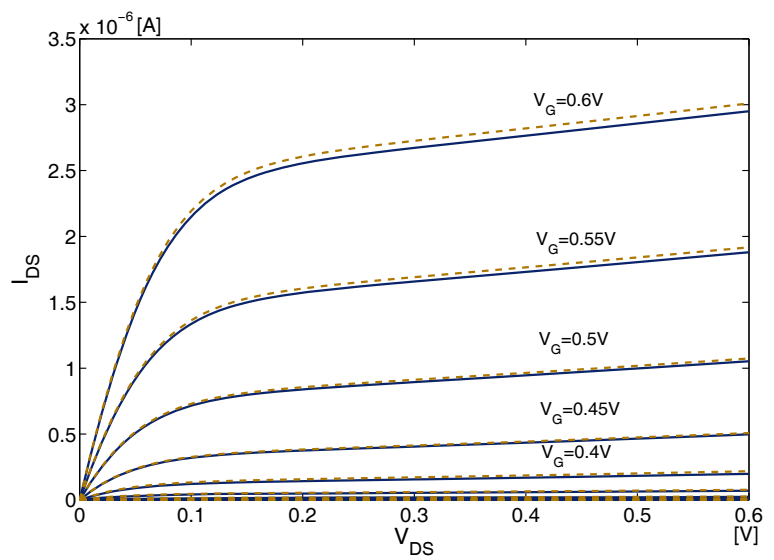

Fig. 9. Drain current characteristics at $T=450 \mathrm{~K}$ and $E_{F}=-0.5 \mathrm{eV}$ for FETToy(solid lines) and piece-wise approximation using Model 2 (dashed lines).

\section{CONCLUSION}

We have proposed a new, fast numerical approach to CNT modelling suitable for a direct implementation in SPICElike circuit simulators. Results provide further evidence to support recent suggestions [8], [10] that numerical integration and Newton-Raphson iterations can be avoided, leading to a substantial acceleration in the model evaluation. Two specific

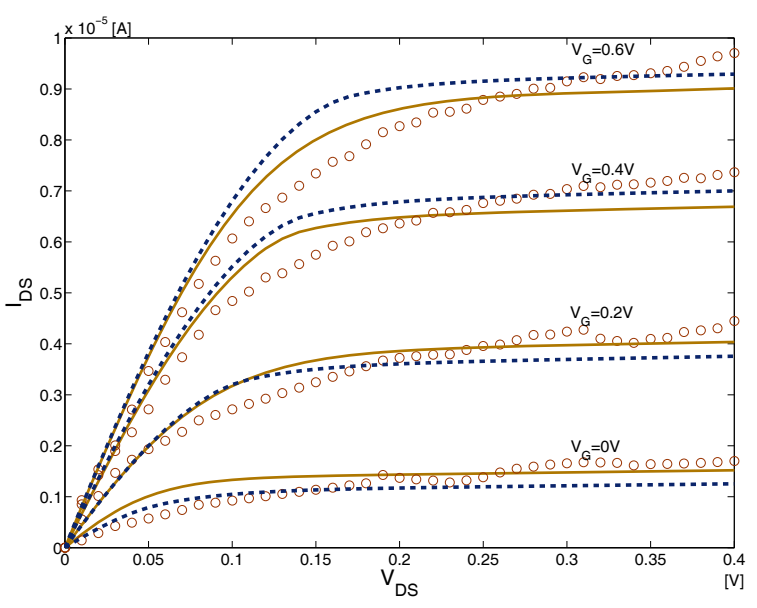

Fig. 10. Comparison with experimental results (circlet lines) of the FETToy model(solid lines) and the proposed model 1 (dashed lines) for $d=1.6 \mathrm{~nm}$, $t_{o x}=50 \mathrm{~nm}, \mathrm{~T}=300 \mathrm{~K}$ and $E_{F}=-0.05 \mathrm{eV}$.

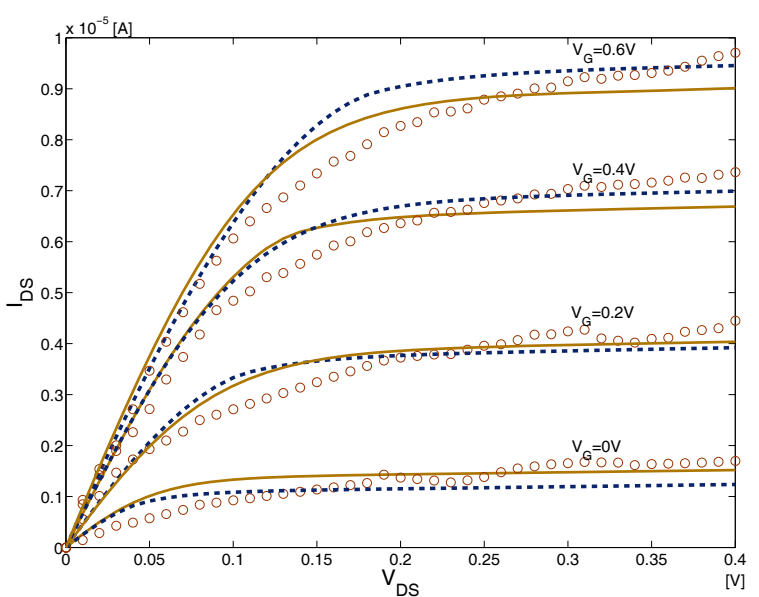

Fig. 11. Comparison with experimental results (circlet lines) of the FETToy model(solid lines) and the proposed model 2(dashed lines) for $d=1.6 \mathrm{~nm}$, $t_{o x}=50 \mathrm{~nm}, \mathrm{~T}=300 \mathrm{~K}$ and $E_{F}=-0.05 \mathrm{eV}$.

piece-wise non-linear approximations to the non-equilibrium mobile charge densities have been developed to enable a closed-form solution of the self-consistent voltage equation and thus eliminate the need for costly iterations. The parameters of the piece-wise ranges assure the continuity of the first derivative and were optimised for fitting accuracy. When compared with FETToy [9], a reference theoretical CNT model, we have demonstrated that the proposed approximation approach leads to a computational cost saving of more than three orders of magnitues while maintaining the modeling accuracy comparable with that of the state-of-the art ballistic transport theory. This research contributes towards the recent efforts in CNT model development for nano-electronic circuit design. These efforts aim to enable practical and efficient simulations of future analogue and digital circuits built using large numbers of CNT devices. Future work will involve extension 
TABLE II

AVErage RMS ERrors in $I_{D S}$ APPRoximation of Model 1 ANd Model 2 For $E_{F}=-0.32 e V$.

\begin{tabular}{|l|c|c|c|c|c|c|}
\hline & \multicolumn{2}{|c|}{ 150K } & \multicolumn{2}{c|}{$300 \mathrm{~K}$} & \multicolumn{2}{c|}{$450 \mathrm{~K}$} \\
\hline$V_{G}[V]$ & Model 1 & Model 2 & Model 1 & Model 2 & Model 1 & Model 2 \\
\hline \hline 0.1 & $4.0 \%$ & $1.7 \%$ & $4.4 \%$ & $2.0 \%$ & $4.6 \%$ & $2.3 \%$ \\
\hline 0.2 & $3.2 \%$ & $1.3 \%$ & $3.6 \%$ & $1.5 \%$ & $3.7 \%$ & $1.9 \%$ \\
\hline 0.3 & $2.5 \%$ & $1.0 \%$ & $2.7 \%$ & $1.0 \%$ & $2.9 \%$ & $1.4 \%$ \\
\hline 0.4 & $1.8 \%$ & $0.7 \%$ & $1.9 \%$ & $0.6 \%$ & $2.3 \%$ & $1.0 \%$ \\
\hline 0.5 & $1.5 \%$ & $0.4 \%$ & $1.6 \%$ & $0.5 \%$ & $2.6 \%$ & $1.1 \%$ \\
\hline 0.6 & $1.9 \%$ & $0.8 \%$ & $2.2 \%$ & $0.9 \%$ & $2.7 \%$ & $1.3 \%$ \\
\hline
\end{tabular}

TABLE III

AVErage RMS ERrors in $I_{D S}$ APPRoXimation of Model 1 AND Model 2 For $E_{F}=-0.5 \mathrm{eV}$.

\begin{tabular}{|l|c|c|c|c|c|c|}
\hline & \multicolumn{2}{|c|}{$150 \mathrm{~K}$} & \multicolumn{2}{c|}{$300 \mathrm{~K}$} & \multicolumn{2}{c|}{ 450K } \\
\hline$V_{G}[V]$ & Model 1 & Model 2 & Model 1 & Model 2 & Model 1 & Model 2 \\
\hline \hline 0.1 & $4.4 \%$ & $2.1 \%$ & $4.7 \%$ & $2.3 \%$ & $4.8 \%$ & $2.8 \%$ \\
\hline 0.2 & $4.0 \%$ & $1.5 \%$ & $4.2 \%$ & $1.8 \%$ & $4.3 \%$ & $2.4 \%$ \\
\hline 0.3 & $3.1 \%$ & $1.2 \%$ & $3.3 \%$ & $1.3 \%$ & $3.5 \%$ & $1.7 \%$ \\
\hline 0.4 & $2.2 \%$ & $1.0 \%$ & $2.9 \%$ & $0.8 \%$ & $2.7 \%$ & $1.4 \%$ \\
\hline 0.5 & $1.8 \%$ & $0.7 \%$ & $2.6 \%$ & $0.8 \%$ & $3.5 \%$ & $1.2 \%$ \\
\hline 0.6 & $2.4 \%$ & $0.9 \%$ & $3.3 \%$ & $1.3 \%$ & $4.1 \%$ & $1.6 \%$ \\
\hline
\end{tabular}

TABLE IV

AVERAge RMS ERRors in $I_{D S}$ APPROXIMATION OF MOdel 1 AND Model 2 For $E_{F}=0 e V$.

\begin{tabular}{|l|c|c|c|c|c|c|}
\hline & \multicolumn{2}{|c|}{$150 \mathrm{~K}$} & \multicolumn{2}{c|}{$300 \mathrm{~K}$} & \multicolumn{2}{c|}{$450 \mathrm{~K}$} \\
\hline$V_{G}[V]$ & Model 1 & Model 2 & Model 1 & Model 2 & Model 1 & Model 2 \\
\hline \hline 0.1 & $2.9 \%$ & $1.6 \%$ & $3.4 \%$ & $1.9 \%$ & $4.0 \%$ & $2.1 \%$ \\
\hline 0.2 & $2.1 \%$ & $1.3 \%$ & $2.6 \%$ & $1.4 \%$ & $3.2 \%$ & $1.7 \%$ \\
\hline 0.3 & $1.6 \%$ & $1.0 \%$ & $2.0 \%$ & $1.1 \%$ & $2.6 \%$ & $1.4 \%$ \\
\hline 0.4 & $1.3 \%$ & $0.6 \%$ & $1.8 \%$ & $0.7 \%$ & $2.3 \%$ & $1.0 \%$ \\
\hline 0.5 & $1.2 \%$ & $0.4 \%$ & $1.3 \%$ & $0.5 \%$ & $2.1 \%$ & $0.8 \%$ \\
\hline 0.6 & $1.5 \%$ & $0.7 \%$ & $2.0 \%$ & $0.7 \%$ & $3.1 \%$ & $1.0 \%$ \\
\hline
\end{tabular}

of the model to include non-ballistic transport effects as well as further testing for speed and modelling accuracy in practical logic circuit structures based on CNT devices. To facilitate future CNT circuit simulations we have developed a VHDLAMS implementation of Model 2 and made it available for public use through the Southampton VHDL-AMS Validation Suite website [14].

\section{ACKNOWLEDGEMENT}

The authors would like to acknowledge the support of EPSRC/UK for funding this project in part under grant $\mathrm{EP} / \mathrm{E} 035965 / 1$.

\section{REFERENCES}

[1] Anisur Rahman, Jing Guo, Supriyo Datta, and Mark S. Lundstrom. Theory of ballistic nanotransistors. Electron Devices, IEEE, 50(9):18531864, September 2003.

[2] Phaedon Avouris, Joerg Appenzeller, Richard Martel, and Shalom J. Wind. Carbon nanotube electronics. Proceedings of the IEEE, 91(11):1772-84, November 2003.

[3] Arash Hazeghi, Tejas Krishnamohan, and H.-S. Philip Wong. SchottkyBarrier Carbon Nanotube Field-Effect Transistor Modeling. In Sixth IEEE Conference on Electron Devices, volume 54, Lausanne, Switzerland, March 2007.

[4] Thao Dang, Lorena Anghel, and Regis Leveugle. CNTFET basics and simulation. In IEEE Int. conf. on Design and Test of Integrated Systems in Nanoscale Technology (DTIS), Tunis, Tunisia, 5-7 September 2006.
[5] Chris Dwyer, Moky Cheung, and Daniel J. Sorin. Semi-empirical SPICE models for carbon nanotube FET logic. In 4th IEEE Conference on Nanotechnology, Munich, Germany, 16-19 Aug. 2004.

[6] Arijit Raychowdhury, Saibal Mukhopadhyay, and Kaushik Roy. A circuit-compatible model of ballistic carbon nanotube field-effect transistors. Applied Physics Letters, 23(10):1411-20, October 2004.

[7] Bipul C. Paul, Shinobu Fujita, Masaki Okajima, and Thomas Lee. Modeling and analysis of circuit performance of ballistic CNFET. In 2006 Design Automation Conference, San Francisco, CA, USA, 24-28 July 2006.

[8] Hamidreza Hashempour and Fabrizio Lombardi. An efficient and symbolic model for charge densities in ballistic carbon nanotube FETs. IEEE-NANO, 1:17-20, 2006.

[9] Anisur Rahman, Jing Wang, Jing Guo, Sayed Hasan, Yang Liu, Akira Matsudaira, Shaikh S. Ahmed, Supriyo Datta, and Mark Lundstrom. Fettoy 2.0 - on line tool, 14 February 2006. https://www.nanohub.org/ resources $/ 220 /$.

[10] Tom J. Kazmierski, Dafeng Zhou, and Bashir M. Al-Hashimi. A fast, numerical circuit-level model of carbon nanotube transistor. In IEEE Int. Workshop on Design and Test of Defect-Tolerant Nanoscale Architectures (Nanoarch), Santa Clara, CA, 21-22 October 2007.

[11] Ming-Hsun Yang, Kenneth B. K. Teo, Laurent Gangloff, William I. Milne, David G. Hasko, Yves Robert, and Pierre Legagneux. Advantages of top-gate, high-k dielectric carbon nanotube field-effect transistors. Applied Physics Letters, 88(11):113507-1-3, March 2006.

[12] Paul L. McEuen, Michael S. Fuhrer, and Hongkun Park. Single-walled carbon nanotube electronics. Nanotechnology, IEEE Transactions, 1(1):78-845, March 2002.

[13] Ali Javey, Ryan Tu, Damon Farmer, Jing Guo, Roy Gordon, and Hongjie Dail. High performance n-type carbon nanotube field-effect transistors with chemically doped contacts. Nano Letters, 5:345-348, 2005.

[14] Southampton VHDL-AMS Validation Suite, 2007. https://www.syssim. ecs.soton.ac.uk/index.htm. 